\title{
THE 2008 CONGENITAL CYTOMEgaloviruS CONFERENCE, 5-7 November, Centers for Disease Control and Prevention, Atlanta
}

\author{
A Vossen (A.C.T.M.Vossen@lumc.nl) ${ }^{1}$, J de Vries $^{1}$, B van der Zeijst ${ }^{1}$ \\ 1. Department of Medical Microbiology, Leiden University Medical Center, Leiden, the Netherlands
}

The theme of this conference was "public health action towards awareness, prevention, and treatment". The purpose was to bring together researchers and clinicians from various fields to discuss the latest research on congenital cytomegalovirus (CMV) infection and how these findings can be translated into public health action for better health of women and children. In addition, families with children affected by congenital CMV participated in the conference, either in integrated sessions together with the experts or in separate sessions only for the families. These children were a testimony of the severe disabilities that congenital infections can cause.

More than 250 participants from all over the world attended the conference, which included about 50 oral presentations and 50 poster presentations. In this report the different topics of this conference will be briefly discussed, with a focus on disease burden and public health. Most presentations can be found at: http://www.congenitalcmv.org/cmvslides2008.htm

\section{Epidemiology}

Michael J. Cannon (United States (US) Centers for Disease Control and Prevention (CDC)), one of the organisers of this conference, described three areas of recent epidemiologic studies at the CDC.

- CMV awareness among women and obstetrician/gynaecologists,

- Seroprevalence data leading to an understanding of transmission modes on a population level,

- Studies on the overall burden of congenital CMV infection and disease and the particular burden due to permanent, bilateral hearing loss.

These studies are intended to identify women who are at high risk of giving birth to children with congenital CMV and likely to profit from antiviral treatment or other interventions, and to clarify what messages need to be communicated about congenital CMV prevention. Studies on the prevalence of CMV infections in general show that CMV seropositivity is highly determined by racial/ethnic factors and seropositivity of siblings and mother. CMV infections during pregnancy occur in about eight of 1,000 pregnancies. Most of these congenital CMV infections occur in previously seropositive mothers [1]. Between 17 and $20 \%$ of these congenitally infected children will have permanent disabilities [2].

Karen B. Fowler (Department of Paediatrics, University of Alabama at Birmingham, US) showed that new developments in diagnosing and treating CMV infections, as well as an emerging interest from the US Newborn Hearing Screening Community for the identification of CMV-related hearing loss, has resulted in a need to reconsider surveillance or screening programmes for congenital CMV infection.

Suzanne Luck (Royal Free and University College Medical School, London, United Kingdom (UK)) reported on a newly developed CMV-related treatment registry of pregnant women and infants in the UK that is currently being extended to the rest of the European Union (EU).

\section{Postnatal treatment and follow-up}

The benefits and risks of current antiviral treatments for children with congenital CMV were presented by David Kimberlin (University of Alabama at Birmingham, US). Data on the treatment of congenital CMV are only available for babies that are born symptomatic. In this group, administration of intravenous ganciclovir for six weeks protected against hearing deterioration. Recently, it has been demonstrated that administration of an oral solution of valganciclovir resulted in similar blood concentrations of ganciclovir as intravenous administration of ganciclovir. A new multicenter study conducted by the National Institute of Allergy and Infectious Diseases (NIAID) Collaborative Antiviral Study Group is now evaluating whether six months of oral valganciclovir therapy results in better hearing and neuro-developmental outcomes than six weeks of oral valganciclovir therapy.

The long-term sequelae of congenital CMV on hearing loss and brain development were discussed by John Eichwald (US CDC) and Ira Adams-Chapman (Emory University, Atlanta, US).

\section{Pathogenesis and immunology}

In this session, an up-date was given on the latest insight into the pathogenesis of congenital CMV and the immunological responses to this infection. Lenore Pereira summarised several studies on the pathogenesis of intra-uterine infection and the histopathological effects on the uterine-placental interface, such as villous inflammation, fibrosis and necrosis. Immunostaining revealed expression of proteins associated with hypoxia. These results suggest direct viral damage resulting in placental hypoxia. She also showed that treatment with intravenous hyperimmunoglobulin, a recently reported intervention in women with primary CMV infection, resulted in compensatory vascularisation of the placenta and villous 
regeneration. Another presentation showed that a murine model that had previously been used to study the pathogenesis of CMV infection has now been used to study hearing loss.

\section{Awareness and behavioural interventions}

The main message of this session was that only few women have heard of congenital CMV, and although studies have shown that prevention is possible by adopting certain hygienic behaviours, most women were not informed by their obstetricians or gynaecologists about the risks of CMV infection and about possible hygienic measures. Information on the internet regarding CMV prevention is also lacking.

On the other hand, if counselling is applied, women are motivated to be screened for CMV IgG antibodies and to apply hygienic measures.

\section{Prenatal diagnosis, prognostic indicators, correlates of} immunity, and treatment

Maria Grazia Revello (Servizio di Virologia, Fondazione IRCCS Policlinico San Matteo, Pavia, Italy) focused on recent developments in prenatal diagnosis. The main diagnostic methods are immunological detection of infection in the mother, detection of viral DNA and marker proteins in foetuses, and ultrasound examination. These methods have greatly improved the possibility of counselling pregnant women. Not only can infected foetuses at increased risk of congenital disease be identified more reliably, they also allow more efficient monitoring of the effect of newly described interventions, such as CMV hyperimmunoglobulins and valaciclovir.

Recently, administration of hCMV-specific immunoglobulin has been reported to spectacularly reverse the prognosis in severely affected foetuses [1]. Maria Grazia Revello announced that a multicenter randomised, double blind, placebo-controlled trial in pregnant women with primary CMV infection will start in Italy. The trial will concentrate on the prevention of mother-to-child transmission by administration of hCMV-specific immunoglobulin.

A comparable trial was presented by a second speaker, Mara Dinsmoor from Evanston Northwestern Healthcare.

Stuart Adler summarised in his lecture the findings from CMV hyperimmunoglobulin trials and proposed guidelines for treatment and monitoring. More treatment options may be underway, as an orally active analogue of cidofovir was shown to be effective in limiting CMV infection in a guinea pig model.

This session was concluded by an interesting discussion on the advantages and disadvantages of prenatal screening. The general opinion of the experts was that previous obstacles to prenatal screening, such as limited knowledge on the foetal outcome, the lack of reliable prenatal diagnostics and of intervention possibilities, have now been overcome. It is time to consider a well-designed prenatal screening programme.

\section{Vaccines}

For several years now, developing a vaccine for CMV has been regarded as a top public health priority for the US because of the frequency of congenital CMV infection and its impact on sensory, cognitive and motor disability in children. In this session, the many efforts towards vaccine development, the results in animal models and the first results in phase II trials were presented.
Robert Pass (University of Alabama at Birmingham, US) focused on a CMV glycoprotein $B(g B)$ vaccine. A recent phase II clinical trial showed an overall vaccine efficacy of $50 \%$. Rajiv Khanna (Australian Centre for Vaccine Development, Herston, Australia) showed the results of pre-clinical testing of a novel chimeric vaccine based on a replication-deficient adenovirus which encodes, as a contiguous polypeptide, the extracellular domain of the gB protein together with multiple major histocompatibility complex (MHC) class I and II-restricted T cell epitopes of CMV. CMV-specific CD8+ and CD4+ T-cellular as well as humoral immune responses were induced by this vaccine.

All speakers in this session and roundtable discussion emphasised that although pre-clinical and clinical vaccine studies show promising results, many questions still remain to be answered: What do we want to achieve with a vaccine? What will be the target population? What is the best immune correlate of protection? Which animal model can be used?

\section{Newborn screening}

While political and ethical questions remain to be resolved, this session showed that neonatal screening for congenital CMV on dried blood spots (DBS) is technically possible with high sensitivity and in a high throughput fashion. Several speakers emphasised that laboratory testing should be validated thoroughly in order to achieve this high sensitivity. Alternative materials (dried urine or saliva) were shown to be suitable for diagnosing congenital CMV with probably higher sensitivity than DBS testing. However, these materials are currently not usable on a routine basis.

Scott Grosse (National Center on Birth Defects and Developmental Disabilities, US CDC) pointed out that evidence of safe and efficacious treatment is probably crucial if a public health case is to be made for universal screening with DBS.

\section{General conclusions}

Considerable progress has been made in the field of congenital CMV. Knowledge is increasing on virus transmission and pathogenesis of congenital CMV, diagnostic algorithms are designed, and prenatal and postnatal intervention strategies are being evaluated.

However, despite the high disease burden of congenital CMV, public awareness is extremely low. Continued research in this field is needed for the development of preventive and therapeutic strategies that will have a high impact on the quality of life of many children worldwide.

\section{References}

1. Kenneson A, Cannon MJ. Review and meta-analysis of the epidemiology of congenital cytomegalovirus (CMV) infection. Rev Med Virol. 2007;17(4):253-76.

2. Dollard SC, Grosse SD, Ross DS. New estimates of the prevalence of neurological and sensory sequelae and mortality associated with congenital cytomegalovirus infection. Rev Med Virol. 2007;17(5):355-63.

3. Nigro G, Adler SP, La Torre R, Best AM, Congenital Cytomegalovirus Collaborating Group. Passive immunization during pregnancy for congenital cytomegalovirus infection. N Engl J Med. 2005;353(13):1350-62.

This article was published on 5 March 2009.

Citation style for this article: Vossen A, de Vries J, van der Zeijst B. The 2008 congenital cytomegalovirus conference, 5-7 November, Centers for Disease Control and Prevention, Atlanta. Euro Surveill. 2009;14(9):pii=19136. Available online: http:// www.eurosurveillance.org/ViewArticle.aspx?ArticleId=19136 